\title{
Self-assessment and deep learning-based coronavirus detection and medical diagnosis systems for healthcare
}

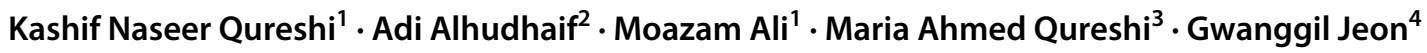

Received: 15 December 2020 / Accepted: 12 August 2021 / Published online: 7 September 2021

(c) The Author(s), under exclusive licence to Springer-Verlag GmbH Germany, part of Springer Nature 2021

\begin{abstract}
Coronavirus is one of the serious threat and challenge for existing healthcare systems. Several prevention methods and precautions have been proposed by medical specialists to treat the virus and secure infected patients. Deep learning methods have been adopted for disease detection, especially for medical image classification. In this paper, we proposed a deep learning-based medical image classification for COVID-19 patients namely deep learning model for coronavirus (DLMCOVID-19). The proposed model improves the medical image classification and optimization for better disease diagnosis. This paper also proposes a mobile application for COVID-19 patient detection using a self-assessment test combined with medical expertise and diagnose and prevent the virus using the online system. The proposed deep learning model is evaluated with existing algorithms where it shows better performance in terms of sensitivity, specificity, and accuracy. Whereas the proposed application also helps to overcome the virus risk and spread.
\end{abstract}

Keywords Coronavirus $\cdot$ Disease $\cdot$ Deep learning $\cdot$ Healthcare $\cdot$ Systems $\cdot$ Challenges $\cdot$ Technologies $\cdot$ Application $\cdot$ Detection · Diagnosis

\section{Introduction}

Coronavirus (COVID-2019) has been replicated all over the world and the cause of several deaths. This virus has started in December 2019 in Wuhan (city of China) and

Gwanggil Jeon

gjeon@inu.ac.kr

Kashif Naseer Qureshi

kashifnq@gmail.com

Adi Alhudhaif

a.alhudhaif@psau.edu.sa

Moazam Ali

muazzam.buic@bahria.edu.pk

Maria Ahmed Qureshi

Drmariaqureshi@gmail.com

1 Department of Computer Science, Bahria University, Islamabad, Pakistan

2 Department of Computer Science, College of Computer Engineering and Sciences in Al-kharj, Prince Sattam bin Abdulaziz University, P.O. Box 151, Al-Kharj 11942, Saudi Arabia

3 University of Catania, Catania, Italy

4 Incheon National University, Incheon, South Korea was rapidly disseminated in all regions [1,2]. All medical health facilities and experts are failed to tackle this virus and it became a challenge for existing healthcare systems. Different types of prevention measures have been introduced to control virus infections such as medications, isolation, and sanitization strategies. Due to the illiteracy rate and shortage of resources in developing countries, these strategies are not working well. The virus also disturbed the economic, educational, industrial, and living systems [2,3]. The middle class and daily wedges community has been suffered more as compared to wealthy peoples. Lockdown is one of the strategy adopted to control the COVID-19 infections. This is not the first experience for human beings because several serious viruses and diseases have been tackled such as malaria, smallpox, and polio. New and integrated methods and technologies have been adopted for data analysis, image classification, and visualize microorganisms [4, 5]. With time, healthcare has controlled these diseases by making vaccines and medications to control and prevent these serious diseases. Some other broad disease control measures are also adopted for COVID-19 control such as sanitization, public awareness, nutrition, and hygiene measures. Figure 1 shows the main process from COVID-19 diagnosis to treatment using deep and machine learning methods. 


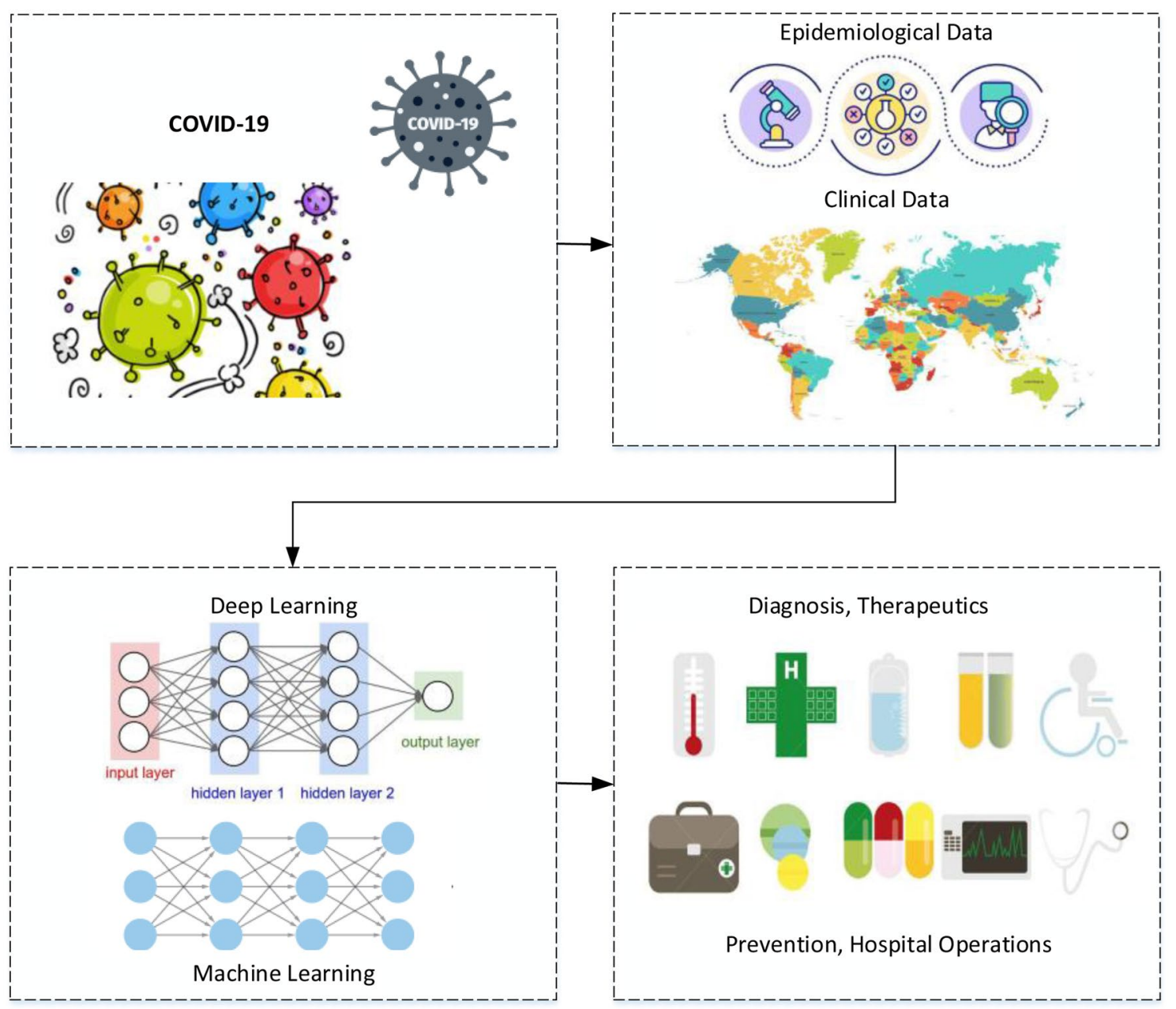

Figure 1. COVID-19 diagnosis to treatment processes

COVID-19 is a respiratory virus and is mostly transmitted with human interaction. This virus is also replicating through human-to-human, droplet, for-oral, and with direct contact. This virus is one of the member of severe acute respiratory syndrome (SARS) and zoonotic pathogen virus family [6]. This virus has been observed in past in the shape of SARS in 2002, with 800 deaths, Middle East respiratory syndrome (MERS-CoV) in 2012 with 860 deaths [7]. According to the last observed numbers from World Health Organization (WHO), COVID-2019 is one of the serious virus which infected around 1,696,588 confirmed cases in six regions, and more than 4105,952 deaths have been reported [8]. In some regions like Europe is the peak region where around 880,106 confirmed cases are reported. Different medicine companies announced that the COVID-19 vaccine will come in near future and many trials have been initiated in different countries [9]. Till now the only solutions are raising awareness, care, isolation, and sanitization. The virus is an electron microscope organ, that is around $60 \mathrm{~nm}-140 \mathrm{~nm}$ in diameter and looks like a crown [10]. The common symptoms of this virus are cough, headache, fever, fatigue, breathlessness, and myalgia. High-risk patients especially old age people have mostly suffered from this virus due to pneumonia and respiratory failure and cause of death. Various virus treatments have been presented such as maintaining physical distance, maintaining nutrition and hydration, and controlling the cough and fever issues. The serious ill people are treated by providing oxygen and nasal prongs, nasal cannula, or ventilators. Due to the shortage of ventilators, the situation is too worst, especially in developing countries. Plasma therapy is also recommended where the plasma is extracted from positive recovered patients and transplanted in infected patients $[11,12]$. However, these treatments are not recommended due to complex transplanted processes.

New and smart technologies are used to diagnose and detect diseases and changed the traditional methods in healthcare systems. The question is how these new technologies handle any new type of medical emergency like the recent coronavirus. Also, how the healthcare systems 
tackle, control and monitor the disease especially which are spread by human-to-human transmissions such as flu, corona, and other types of infections [13, 14]. Although the primary objective is to control the spreading virus using any means which are beneficial for human beings. Deep and machine learning methods are used for data gathering to disease diagnosis, detection, and prediction $[15,16]$. Deep learning methods have gained popularity due to their improved performance and better results. The most common deep learning methods are recurrent neural networks (RNN), bidirectional recurrent neural networks (Bi-LSTM), long short-term memory (LSTM), variational auto-encoder (VAE), and gated recurrent unit (GRU) [17]. These methods are used to solve complex problems using learning from simple depictions. These methods are capable to learn the exact learning property of the data in a deep manner and also utilized multiple layers sequentially. Deep learning methods are widely used in health systems such as drug discovery, biomedicine, and medical image analysis [18]. Deep learning methods are also used to detect the COVID-19 and predict the disease for reference. These methods are used for X-ray image classification or segmentation for feature extraction. The early detection and diagnosis of COVID-19 are very helpful. However, these deep learning methods are not explored well especially for COVID-19.

In this paper, we discuss the COVID-19 in detail and existing literature to handle this virus. This paper proposes a deep learning method namely DLM-COVID-19 for MRI image classification and optimization to detect the disease and improve the existing healthcare systems. Paper also proposes a mobile based application for self-assessment of COVID-19 patients. The main objectives of this paper are as follows:

- Discuss the existing deep learning methods to tackle the COVID-2019.

- Proposed a self-assessment based online system for COVID-19 patients

- Proposed a deep learning model to detect the COVID19 and improve the existing healthcare systems.

- Conclude the paper with possible suggestions to control the COVID-2019

The rest of the paper is organized as follows: Sect. 2 illustrates the related work until now to deal with COVID2019. Section 3 presents the proposed self-assessmentbased model to tackle the COVID-2019. Section 4 presents the deep learning-based model and its design phases. Section 5 presents the experimental results to evaluate the proposed model and its comparison with existing solutions. The paper concludes in Sect. 6 with future directions.

\section{Related work}

Authors in [19] presented a classifier for COVID-19 disease and also explored the relationship between medical images. The authors used a single-center study and applied it to infected patients and categorized them into three types including mild, common, and severe patients. The quantitative analysis is performed by summing up acute lung inflammatory lesions. The severity score is set by summing up the five lobe scores and compared it for clinical classification. After conducting the experiments on patients and observed, that the mild level patients are relatively high where the CT tests are not suitable and feasible as an independent screening tool. The results indicated that the visual quantitative analysis is high and reflects on the COVID-19 classification. In another study [20], the authors used chest CT scans of COVID-19 patients and identified the most common findings. The common findings from images are used to evaluate the disease and its impact on patients' chests. This analysis is based on the radiologist's opinion to identified the new cases. However, this type of strategy has a limited scope and not applicable for big data analysis. Also, the radiologist's opinion varies due to its experience, CT image quality, and availability of comparison images. Some other advanced methods are discussed like artificial intelligence (AI) for diagnosis and control the COVID-19 disease and help the medical experts.

In [21], authors proposed a deep learning method to assist the radiologist for fast identification of CT images. The authors used different patient data collected from hospitals and trained the multi-view fusion model using deep learning and screen patients with COVID-19. This model achieved accuracy, sensitivity, and specificity. Authors in [22], proposed a diagnosis method for COVID-19 using the AI method on CT slices and ten conventional neural network models. These experiments are conducted for diagnosis and achieved the best performance. This study also determined the accuracy, specificity, and sensitivity and recognized that RestNet-101 is recognized as a COVID-19 infection with high sensitivity. In another study [23], the authors designed an AI-based system for COVID-19 diagnosis and quantitative measurement using computed tomography. For medical image analysis, there are some other machine learning methods are designed for classification. The preprocessing methods and hand-crafted methods have been applied using support vector machine (SVM), histogram equalization, and morphological filtering of images. Some other methods are also adopted like ANN, linear dependent analysis (LDA), self-organizing map (SOM). Deep learning methods are adopted especially when complex data processing 
is needed where machine learning failed. Deep learning is not dependent on human intervention and contains numerous layers for a different interpretation of data. Authors in [24], proposed a segmentation model for brain tumor segmentation. This model uses the deep learning CNN method to trained and learn the mapping from MRI brain images and find the space of tumor. This model achieved better segmentation results compared with traditional deep learning CNN networks and SVM classifiers.

Authors in [25], established an early screening model for COVID-19 using pulmonary CT images and deep learning methods. The virus-infected patient's data is collected from China and applied data on different deep learning methods to extract the desired regions. The infection type and overall confidence score for every CT image are analyzed to check the accuracy score. Authors in [26] proposed a model to identified the COVID-19 patients using their chest CT scan images. The deep learning-based diagnostic system is developed to identifying the COVID-19 patients. The proposed model is capable to discriminate the infected patients and localized the lesion features.

\subsection{Discussion}

Another health emergency in the shape of COVID-19 has been started and now it is a global concern. The new variants of this virus have been observed all over the world where the symptoms are different. It is also observed that the infected patients are not diagnosed promptly and carrier of virus. Infected patients are evaluated in terms of their tests, images, CT scans, and other necessary treatments. Lack of resources makes the situation more serious in terms of medical facilities, hospitals, and doctors. The collected samples of images need proper classification and optimization for better diagnosis. The new technologies need to adopt for patient's facilitations without patients' physical appearance in hospital. As discussed, many studies have used deep and machine learning methods for classification using different images of patients. Still, there is a need to design a more attractive user-friendly application and prediction system for COVID-19 patients for better self-assessment and diagnosis.

\section{Proposed COVID-19 detection model}

Diagnostic virology has now entered the mainstream of medical practice. Several methods are being used to diagnose and detect the virus but all those methods are manual and require face-to-face monitoring checkups. The utilization of online methods to detect the possible presence of COVID19 in the subject through a self-assessment test combined with the expertise of a healthcare professional to detect the virus and diagnose it through online-based treatment will decrease the risk of virus spread. The proposed detection system allows the patient to visit the online system and attempt questions, then generates results about the patient current health position based on results. If the patient needs to visit the hospital, the proposed system guides the patients and significantly decreases the risk of virus spread.

The proposed system is based on a model view controller (MVC) architecture which is a secure architecture where the user interacts with a rich graphical user interface layer. It deals with data and controller and acts like the middle man where all the logic is going to fetch the data from the model. The proposed system front end has four modules Self-assessment test, report generation, doctor review, and online medicine facilities. The system offers the user COVID-19 selfassessment test and report generated based on the score of the report. If the score is more than the specified range, it is conveyed to doctors and initiates the final verdict regarding the patient for prescription or some medications.

\subsection{System design}

We used VS CODE (open source code), PHP language, Python, React, Laravel, and other programming languages to design the system. Laravel is used to develop the web application following the MVC model view controller architecture. Laravel gives us features like a modular package system with a dedicated dependency manager, different ways of accessing database utilities, and it is oriented towards syntactic sugar. Self-assessment is a detailed form that appears and has 130 symptoms related to COVID-19 for selection. The report with all symptoms selected by the patient or user will be submitted to the doctor. Figure 2 shows the GUI of the system whereas Fig. 3 shows the working steps of the proposed system.

The GUI interface of the proposed system clearly shows the website for visitors and the registration process for the self-assessment test. There are multiple matches related to COVID-19 that are store in the database to check the patient infection. After this self-analysis, the system generates the report and sends the report for further examination to the doctor or medical systems.

\section{Proposed deep learning solution for COVID-19}

This section presents the best possible deep learning-based solution to control the COVID-2019 namely deep learning model for coronavirus (DLM-COVID-19). The proposed model provides clear and better visual contents using magnetic resource images (MRI) for COVID-19 patients. The COVID-19 infected patients have various abnormalities 


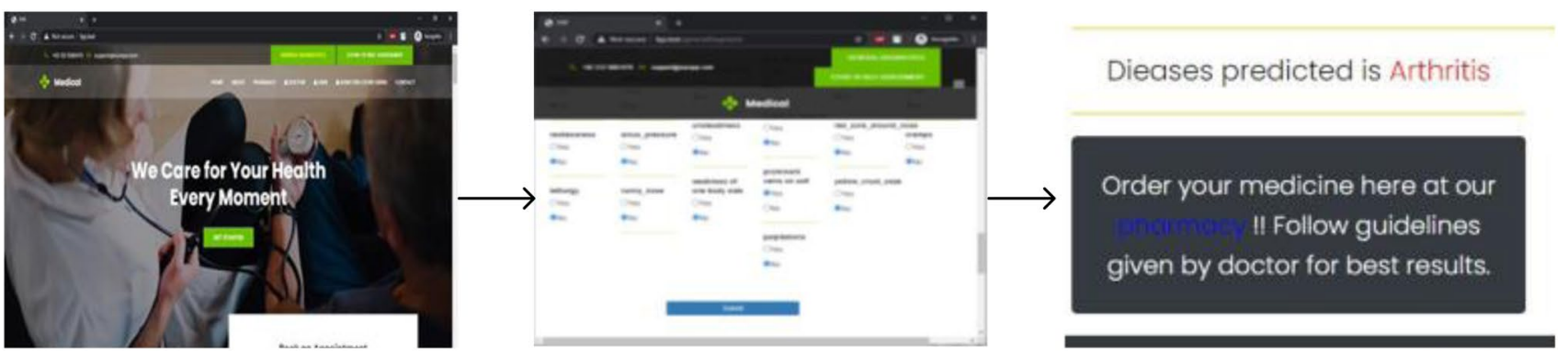

Figure 2. GUI of the proposed system

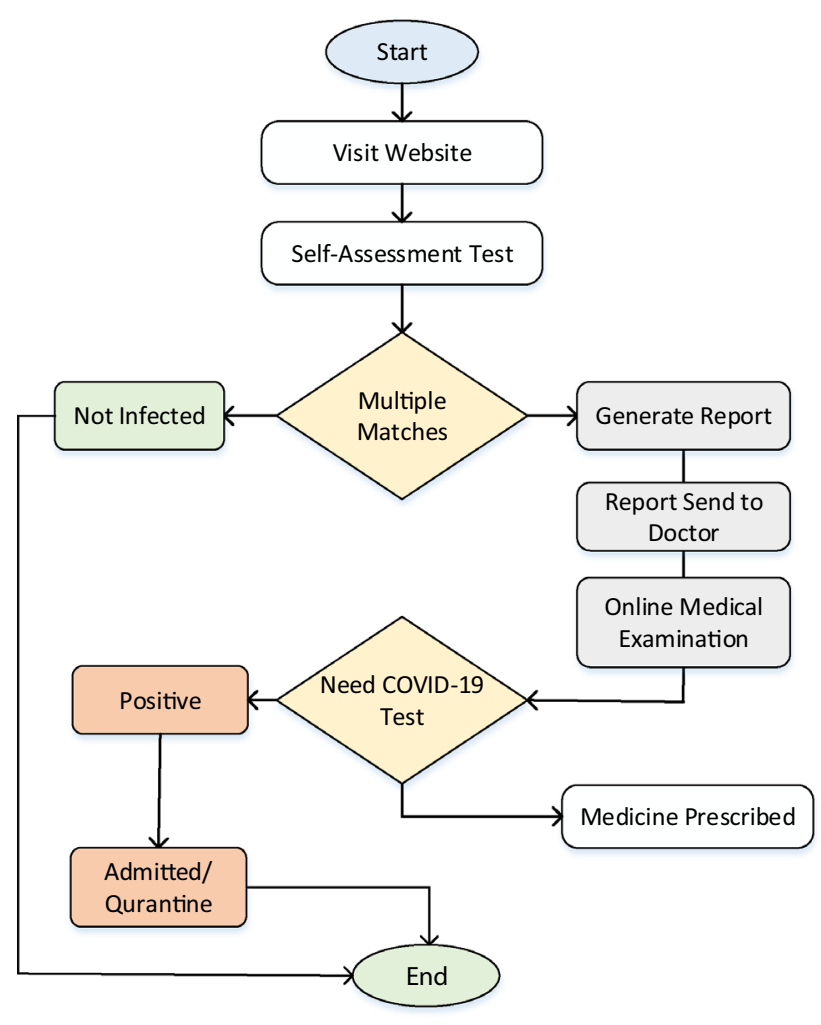

Figure 3. Proposed self-assessment test system

in the brain. The basic purpose of DLM-COVID-19 is to extract the MRI neuroimaging findings with severe COVID-19 infections because of the complex structure of the brain. Feature extraction is also one of the complex process due to radio waves and the detailed internal structure of the brain. The DLM-COVID-19 model improves the quality of MRI images using the clustering method for image segmentation. The proposed model is based on different phases including data collection for input, preprocessing for noise removal, inversion recovery, and enhancement. Figure 4 shows the steps involved in the proposed DLM-COVID-19 model.

\subsection{Data collection}

For data input, we need MRI images especially for those patients who suffered from COVID-19 disease. This task is difficult because of the unavailability of MRI images where the microstructure changes in the central nervous system after virus infection. For this step, we used SARS-CoV-2 data where we used around 50 recovered patients and distributed them into control and non-control categories. The data are collected from different hospitals with the help of medical experts.

\subsection{Data preprocessing}

In the second preprocessing phase, the MRI images are enhanced for detection of the unwanted and suspicious regions in images using the inversion recovery technique and RGB to gray conversion performed. In images, the corrupted factors and noise reduce image accuracy. In the image enhancement phase, the image improves in terms of its contrast level for easily distinguishing the objects. After enhancement, the inversion recovery initiates to reduce the insensitive and magnetic field. The Gaussian high pass filter is utilized for noise reduction and sharpening the contrast. Histogram matching, non-brain tissue stripping, and field correction are also used for the preprocessing $[27,28]$.

\subsection{Data augmentation}

The data augmentation phase is used due to a limited number of training data available. We applied image rotation for increasing the training data. The images are rotated in 90 , 180 , and 270 degrees. This phase is useful and overcomes the overfitting.

\subsection{Learning algorithms}

Convolutional neural networks (CNN) are used for clustering and grouping the images for object recognition. This method also detected the patterns where it classifies the 
Figure 4. Proposed model DLM-COVID-19 steps

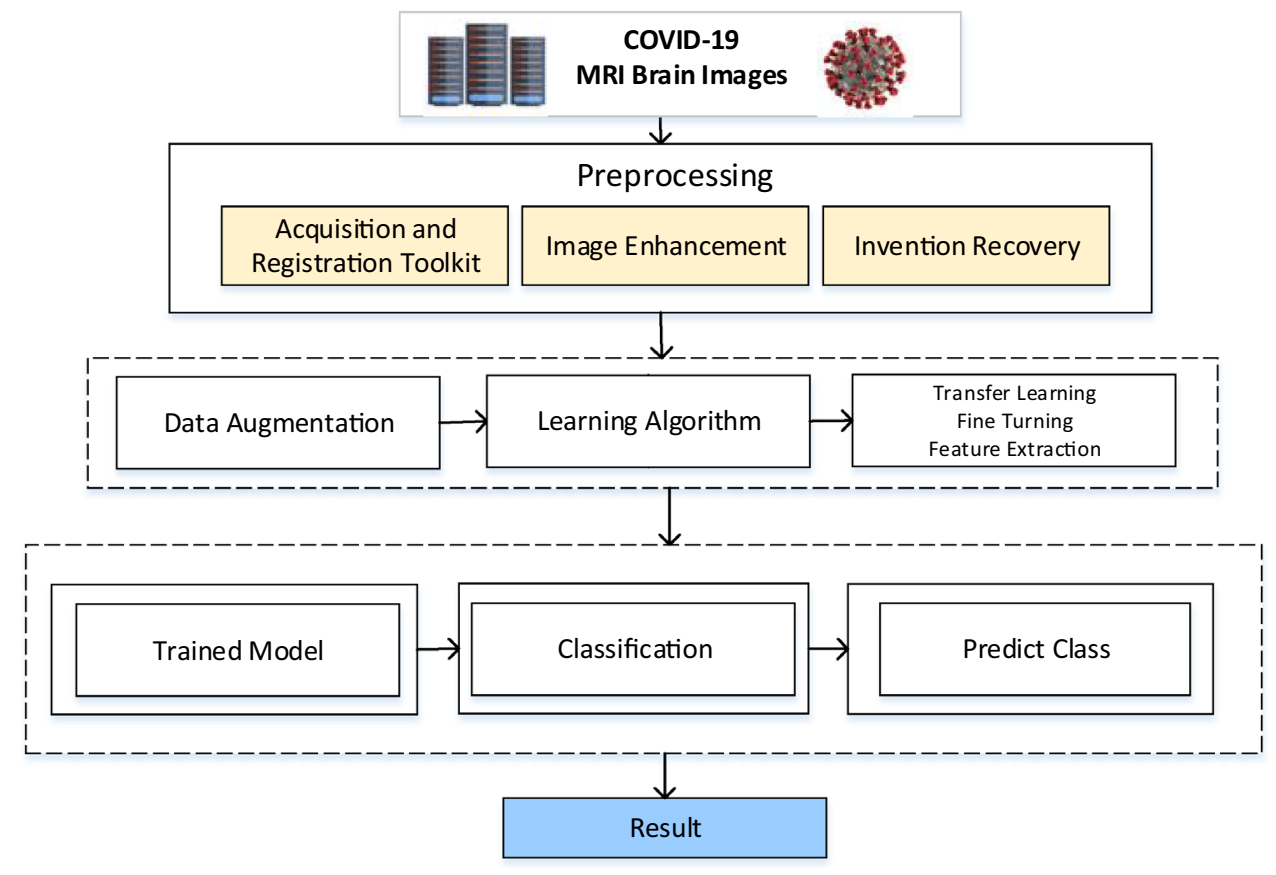

images for feature extraction. CNN has many layers and every layer exposes the attributes or components of the image. CNN contains an input layer, an output layer, and invisible layers between the input and output layer. Convolution input in the image is using convolutional filter and extracted certain features from the image. A rectified linear unit is another powerful layer and it maps negative qualities to zero and keeping up positive qualities. The pooling layer uses rearrangement and output using executing non-linear downsampling.

\subsection{Transfer learning}

Transfer learning is used when a data unavailability exists or not enough data available. This method uses feature extraction and fine-tuning where the transfer of the knowledge gained on one dataset and transfer to another dataset. Finetuning is adopted to tackle the around time and resources issues in deep neural networks, especially for large datasets. The fine-tuning is applying pertained weights during the training process. For fine-tuning, we replaced the last output layer and learn another output layer.

Feature extraction uses CNN which is trained on the dataset. The feature extraction and reduction are applying using Gabor and GLCM filters to extract the features from segments. This phase extracts texture, shape, and intensities. The PCA method is used to eliminate the number of features for accurate classification. The main objective of feature extraction is to decreasing the data using the measurement of certain properties.

\subsection{Classifier training}

$\mathrm{CNN}$ is used for classifier training and check the accuracy of each algorithm and found that SVM has more accuracy for this study. The training time is also shorter compared to other algorithms. First, transforms the input space up to a higher dimensional feature space using a non-linear mapping method and then constructs the separate hyperplane with maximum distance close to the training set. The learning algorithm analyzes the data for regression and classification. This algorithm trains on training data and the trained model then classify the test data. The SVM algorithm can only have trained on labeled data and it cannot operate on unlabeled data or unsupervised data. After this process, the next step is segmentation on test datasets. Segmentation is issued in MRI image division into patchwork regions where each part is homogenous in some sense. Segmentation is one of the method for diagnosis the objects from MRI images [29]. All voxels have passed into SVM and then turn their output where the probabilities of the voxel are related to six classes. The voxel has been assigned to the class which has the highest probability. Networks are trained with six label classes. Six classes are also included for network results. This process provides a clear contrast between soft tissues and makes more clear the texture of organs. Segmentation is the initial step applied to MRI images especially for low-level images where the processing will apply to transform the greyscale or color image into a high-level image. The segmented MRI image where gray matter (GM), white matter (WM), air, and ventricles are observed. 


\subsection{Classification}

For the data classification, the proposed model used support vector machine (SVM) and principle component analysis (PCA). The SVM is used for discriminative classification by separating the hyperplane, and SVM provides supervised learning for classification and regression tasks which generates non-overlapping partitions and usually employs all attributes. We also used the PCA method for reduction. In the PCA classification method, we reduce the dimensionality of the data set and make a correlation of variables with each other. The PCA process also tests the MRI images and is identified by projecting the image into the Eigenspace to gain the corresponding set of weights and then compared the set of weights of the faces in training set. Low dimensional feature issue can be examined where Let $\mathrm{B}=(\mathrm{B} 1, \mathrm{~B} 2, \mathrm{~B} 3, \mathrm{~B} 4, \ldots \ldots \ldots \mathrm{Bi} \ldots \ldots \ldots \mathrm{Bn})$ denotes the $\mathrm{n} \times \mathrm{X}$ data matrix and $\mathrm{B} 1$ is a face vector of dimension $\mathrm{n}$, where $\mathrm{n}$ denotes the total pixel of MRI image and $\mathrm{X}$ is the face images in the training set. The PCA method is used for linear transformation from the original image vector to a projection feature vector.

$A=C^{T} B$

In the above Eq. 1, the A presents the $\mathrm{d} \times \mathrm{X}$ feature matrix, $\mathrm{d}$ denotes the dimension of feature vector and $\mathrm{C}$ is transformation matrix and $\mathrm{n} \times \mathrm{d}$ is transformation matrix where columns are the eigenvectors corresponding with the d using Eq. 2.

$\nabla e i=I e i$

In the above equation, the $\nabla$ denotes the eigenvectors values of the matrix where the total number of scatter matrix I and the mean image is identified as

$I=\sum_{i=1}^{x}(B i-\mu)(B i-\mu)^{T}, \mu=1 / X \sum_{i=1}^{x} B i$

After analyzing the linear transformation $C^{T}$ the scatter of the transformed feature vectors $\{\mathrm{D} 1, \mathrm{D} 2, \ldots \ldots \mathrm{DX}\} \mathrm{D}$ is $C^{T}$ IC. In the PCA method, the projection $C_{o p t}$ is selected to maximizing the determinant of total scatter of the projected sample as presents in Eq. 4.

$C_{\text {opt }}=\arg ^{\max -w}\left|C^{T} I C\right|=\left[D_{1}, D_{2} \ldots \ldots \ldots D_{m}\right]$

In above Eq. 4, the $\{c, l i=1,2 \ldots \ldots \ldots D\}$ is the set of $\mathrm{n}$-dimensional eigenvectors of I corresponding to the D largest Eigen values. In addition, the input vector or face vector in an n-dimensional space is decreased to a feature vector in a D-dimensional subspace.
Table 1 Training and test images

\begin{tabular}{|c|c|c|c|c|}
\hline \multirow[t]{2}{*}{ Total images } & \multicolumn{2}{|c|}{$\begin{array}{l}\text { Number of images in the } \\
\text { training set }(300)\end{array}$} & \multicolumn{2}{|c|}{$\begin{array}{l}\text { Number of images in } \\
\text { testing set (150) }\end{array}$} \\
\hline & Normal & Abnormal & Normal & Abnorm \\
\hline $\begin{array}{l}300 \text { (images are } \\
\text { very less) }\end{array}$ & 150 & 150 & 75 & 75 \\
\hline
\end{tabular}

Table 2 Comparison analysis

\begin{tabular}{llllll}
\hline $\begin{array}{l}\text { Image } \\
\text { grades }\end{array}$ & $\begin{array}{l}\text { Clas- } \\
\text { sified } \\
\text { images }\end{array}$ & $\begin{array}{l}\text { Miss- } \\
\text { classified } \\
\text { images }\end{array}$ & Accuracy & $\begin{array}{l}\text { Specific- } \\
\text { ity }\end{array}$ & Sensitivity \\
\hline Grade I & 38 & Nil & $98.8 \%$ & $90.4 \%$ & $98.3 \%$ \\
Grade II & 38 & Nil & $97.1 \%$ & $91.2 \%$ & $97.3 \%$ \\
Grade III & 35 & 2 & $96.3 \%$ & $89.2 \%$ & $96.6 \%$ \\
Grade IV & 35 & 2 & $94.4 \%$ & $87.3 \%$ & $93.4 \%$ \\
\hline
\end{tabular}

\section{Experiment results}

In this section, we present different experiments in detail using qualitative image analysis. This analysis is one of the well-known methods in clinical scenarios. The three main performance parameters are selected to analyze the system performance sensitivity, accuracy, and specificity. The false positive, negative, and error rate are also measured. The classification is conducted using the widest performance parameters. Table 1 shows the training and tests COVID-19 patient's image data.

The CNN is adopted to train the model where we have several input layer units, several hidden layers, learning rate, and error rate. First of all, the images are classified into normal and abnormal types as shown in Table 1 . The accuracy is around $98.8 \%$, sensitivity specificity is $90.4 \%$, and sensitivity is $98.3 \%$. All the features have $98 \%$ accuracy using principle component analysis (PCA). The extracted feature components are classified and achieved around $98 \%$ accuracy. Miss-classified images mean where we faced difficulty to learn features for representation. Table 2 presents the classification rate based on true-positive, true-negative, false-positive, and false-negative in terms of sensitivity, specificity, and accuracy.

The proposed DLM-COVID-19 model is compared with state-of-the-art existing models including multiscale fuzzy c-means (MsFCM) [30] and DCNN-F-SVM [24]. These existing models are also designed for MRI brain images for COVID-19. MsFCM is based on PCA and SVM where the DCNN-F-SVM method is based on the deep CNN method. MsFCM uses a diffusion filter to process the MRI images and applied a fuzzy $\mathrm{C}$-mean classification method. For the 
Table 3 Comparison analysis

\begin{tabular}{lllll}
\hline S/No. & Approaches & Accuracy (\%) & Specificity (\%) & Sensitivity (\%) \\
\hline 1 & MsFCM & $85 \%$ & $84 \%$ & $89 \%$ \\
2 & DCNN-F-SVM & NA & $92 \%$ & $91 \%$ \\
3 & Proposed DLM-COVID-19 & $98.4 \%$ & $95 \%$ & $94 \%$ \\
\hline
\end{tabular}

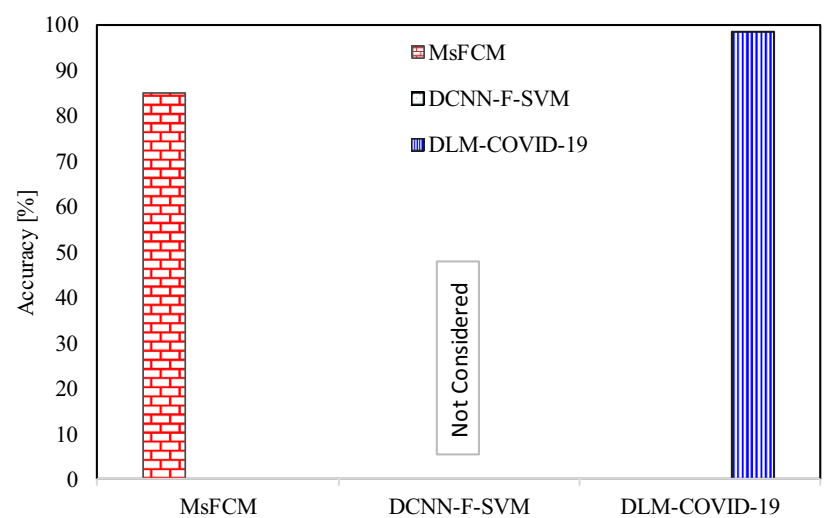

Figure 5. Accuracy of DLM-COVID-19 with existing algorithms

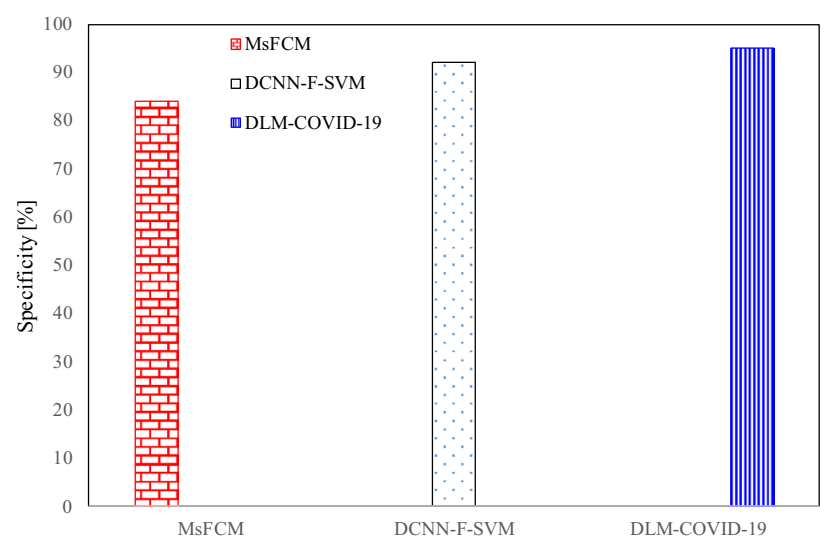

Figure 6. Specificity of DLM-COVID-19 with existing algorithms

comparison, MsFCM on synthesized images with various contrast McGill brain MRI images database are used. On the other hand, the DCNN-F-SVM model has been developed for the segmentation of brain MRI images and predicted the labels from deep CNN training. The main issue in fuzzy mean methods is utilizing high computational resources. The proposed model DLM-COVID-19 is compared with existing algorithms and results are showing in Table 3 and Fig. 5.

Table 3 shows the experimental results obtained using the proposed model trained CNN-based classifier and two existing methods. The proposed model has higher performance in terms of accuracy, specificity, and sensitivity. The results

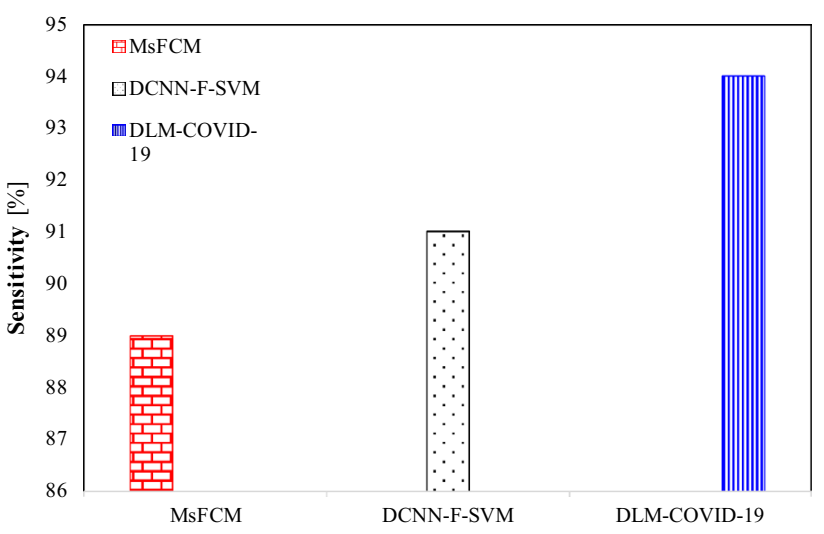

Figure 7. Sensitivity of DLM-COVID-19 with existing algorithms

obtained from the classification process are graphically presenting in Fig. 5, 6, and 7, respectively.

Figure 5 shows the better performance of the proposed model DLM-COVID-19 compared to the existing MsFCM and DCNN-F-SVM methods. The proposed model results are continuous training results. The highest results are achieved with the simple and more efficient design of the proposed model. The impact of accuracy has a significant role in the performance of the algorithm for achieving the more consistent diagnosis of MRI images. The results indicated that the proposed model is better in both environments including test and training images. The proposed model is a rapid, easy to handle, and cost effective solution for COVID19 brain MRI image classification.

Figure 6 shows the comparison of the results of the proposed model with existing methods MsFCM and DCNN-FSVM methods in terms of specificity. The proposed model results have better results compared to MsFCM. The specificity refers to an ability where we identify the true-negative rate with continuous training. On the other hand, the DCNN-F-SVM method also has fewer sensitivity results. But the MsFCM is at $80 \%$ whereas the proposed model has a higher $90 \%$ specificity. The highest results are achieved with the simple and more efficient design of the proposed model. The impact of specificity has a significant role in the performance of an algorithm for achieving the more consistent diagnosis of MRI images. The results indicated that the proposed algorithm is better in both environments including test and training images. The proposed algorithm is a 
rapid, easy to handle and cost effective solution for brain MRI image classification.

Figure 7 shows the comparison of the proposed model with existing MsFCM method in terms of sensitivity. The proposed model results are better compared to MsFCM. The sensitivity refers to the ability in which the test is identifying with a true-positive rate. However, the MsFCM is at $79 \%$ whereas the proposed model is at $87 \%$ which is a good sign of success. The proposed model is more effective and simple in terms of processing and classification. The impact of sensitivity has a broad impact on overall performance for achieving the more consistent diagnosis of MRI images. The results indicated that the proposed model is better in both environments including test and training images. The proposed model is a fast solution, especially for MRI image classification.

\section{Conclusion}

The healthcare systems have been suffered from COVID-19 where the current resources have not fulfilled the human being health demands. This virus has a serious impact on human health, especially on the brain in terms of various abnormalities. These indications are observed from MRI images. However, how to classifying these complex images is still a challenge for medical experts and healthcare systems. This paper presented a deep learning-based model for MRI image classification, feature extraction, and optimization and proposed a self-assessment online platform for COVID-19 detection. Deep learning has gained a lot of popularity, especially for MRI image classification. The proposed model combined with the deep learning model for brain MRI images of COVID-19 patients namely the Deep Learning Model for Coronavirus (DLM-COVID-19). This model improves the MRI image classification and optimization for better diagnosis. This model has achieved better performance especially in the area of brain images which is neglected due to the unavailability of the dataset. COVID19 has a serious impact on the patient brain. The proposed model is helpful to improve the classification and optimization using a nature-inspired algorithm. The proposed model is evaluated with existing algorithms and shows better performance in terms of sensitivity, specificity, and accuracy. In the future, we will use a large dataset to evaluate the proposed model performance.

\section{References}

1. N. Zhu, D. Zhang, W. Wang, X. Li, B. Yang, J. Song, X. Zhao, B. Huang, W. Shi, and R. Lu, A novel coronavirus from patients with pneumonia in China, 2019, New England Journal of Medicine, 2020.

2. H. Alanazi, A. Abdullah, K. Qureshi, and A. Ismail, Accurate and dynamic predictive model for better prediction in medicine and healthcare, Irish Journal of Medical Science (1971), vol. 187, no. 2, pp. 501-513, 2018.

3. Alanazi, H.O., Abdullah, A.H., Qureshi, K.N.: A critical review for developing accurate and dynamic predictive models using machine learning methods in medicine and health care. J. Med. Syst. 41(4), 69 (2017)

4. P. S. Brachman, "Infectious diseases-past, present, and future," ed: Oxford University Press, 2003.

5. Hossain, M.S., Muhammad, G., Guizani, N.: Explainable AI and mass surveillance system-based healthcare framework to combat COVID-I9 like pandemics. IEEE Network 34(4), 126-132 (2020)

6. Yin, Y., Wunderink, R.G.: MERS, SARS and other coronaviruses as causes of pneumonia. Respirology 23(2), 130-137 (2018)

7. Q. Li, X. Guan, P. Wu, X. Wang, L. Zhou, Y. Tong, R. Ren, K. S. Leung, E. H. Lau, and J. Y. Wong, "Early transmission dynamics in Wuhan, China, of novel coronavirus-infected pneumonia," New England Journal of Medicine, 2020.

8. W. H. Organization, "Novel coronavirus (COVID-19) situation," 4 April 2020, Available: https://who.sprinklr.com/.

9. F. Ullah, A. H. Abdullah, M. Q. Jan, and K. N. Qureshi, "Patient data prioritization in the cross-layer designs of wireless body area network," Journal of Computer Networks Communications, vol. $2015,2015$.

10. D. D. Richman, R. J. Whitley, and F. G. Hayden, Clinical virology. John Wiley \& Sons, 2016.

11. Jin, Y.-H., Cai, L., Cheng, Z.-S., Cheng, H., Deng, T., Fan, Y.-P., Fang, C., Huang, D., Huang, L.-Q., Huang, Q.: A rapid advice guideline for the diagnosis and treatment of 2019 novel coronavirus (2019-nCoV) infected pneumonia (standard version). Mil. Med. Res. 7(1), 4 (2020)

12. G. Muhammad, M. S. Hossain, and N. Kumar, EEG-based pathology detection for home health monitoring, IEEE Journal on Selected Areas in Communications, 2020.

13. A. Tayal, J. Gupta, A. Solanki, K. Bisht, A. Nayyar, and M. Masud, DL-CNN-based approach with image processing techniques for the diagnosis of retinal diseases, Multimedia Systems, pp. 1-22, 2021.

14. C. Iwendi, K. Mahboob, Z. Khalid, A. R. Javed, M. Rizwan, and U. Ghosh, Classification of COVID-19 individuals using adaptive neuro-fuzzy inference system, Multimedia Systems, pp. 1-15, 2021.

15. A. Winter, S. Stäubert, D. Ammon, S. Aiche, O. Beyan, V. Bischoff, P. Daumke, S. Decker, G. Funkat, and J. E. Gewehr, Smart medical information technology for healthcare (SMITH), Methods of information in medicine, vol. 57, no. S 01, pp. e92-e105, 2018.

16. A. Zeroual, F. Harrou, A. Dairi, and Y. Sun, Deep learning methods for forecasting COVID-19 time-Series data: A Comparative study, Chaos, Solitons \& Fractals, vol. 140, p. 110121, 2020.

17. S. Wang, B. Kang, J. Ma, X. Zeng, M. Xiao, J. Guo, M. Cai, J. Yang, Y. Li, and X. Meng, A deep learning algorithm using CT images to screen for Corona Virus Disease (COVID-19), MedRxiv, 2020.

18. Hossain, M.S., Muhammad, G.: Deep learning based pathology detection for smart connected healthcares. IEEE Network 34(6), 120-125 (2020)

19. K. Li, Y. Fang, W. Li, C. Pan, P. Qin, Y. Zhong, X. Liu, M. Huang, Y. Liao, and S. Li, CT image visual quantitative evaluation and clinical classification of coronavirus disease (COVID-19), European radiology, pp. 1-10, 2020.

20. Chung, M., Bernheim, A., Mei, X., Zhang, N., Huang, M., Zeng, X., Cui, J., Xu, W., Yang, Y., Fayad, Z.A.: CT imaging features of 
2019 novel coronavirus (2019-nCoV). Radiology 295(1), 202-207 (2020)

21. X. Wu, H. Hui, M. Niu, L. Li, L. Wang, B. He, X. Yang, L. Li, $\mathrm{H}$. Li, and J. Tian, Deep learning-based multi-view fusion model for screening 2019 novel coronavirus pneumonia: a multicentre study, European Journal of Radiology, p. 109041, 2020.

22. A. A. Ardakani, A. R. Kanafi, U. R. Acharya, N. Khadem, and A. Mohammadi, Application of deep learning technique to manage COVID-19 in routine clinical practice using CT images: Results of 10 convolutional neural networks, Computers in Biology and Medicine, p. 103795, 2020.

23. K. Zhang, X. Liu, J. Shen, Z. Li, Y. Sang, X. Wu, Y. Zha, W. Liang, C. Wang, and K. Wang, Clinically applicable AI system for accurate diagnosis, quantitative measurements, and prognosis of covid-19 pneumonia using computed tomography, Cell, 2020.

24. W. Wu, D. Li, J. Du, X. Gao, W. Gu, F. Zhao, X. Feng, and H. Yan, An Intelligent Diagnosis Method of Brain MRI Tumor Segmentation Using Deep Convolutional Neural Network and SVM Algorithm, Computational and Mathematical Methods in Medicine, vol. 2020, 2020.

25. Xu, X., Jiang, X., Ma, C., Du, P., Li, X., Lv, S., Yu, L., Ni, Q., Chen, Y., Su, J.: A deep learning system to screen novel coronavirus disease 2019 pneumonia. Engineering 6(10), 1122-1129 (2020)
26. Y. Song, S. Zheng, L. Li, X. Zhang, X. Zhang, Z. Huang, J. Chen, H. Zhao, Y. Jie, and R. Wang, Deep learning enables accurate diagnosis of novel coronavirus (COVID-19) with CT images, MedRxiv, 2020.

27. T. S. Yoo, M. J. Ackerman, W. E. Lorensen, W. Schroeder, V. Chalana, S. Aylward, D. Metaxas, and R. Whitaker, Engineering and algorithm design for an image processing API: a technical report on ITK-the insight toolkit, Studies in health technology and informatics, pp. 586-592, 2002.

28. C. Wang and Ö. Smedby, Fully automatic brain segmentation using model-guided level sets and skeleton-based models, MIDAS $J$, vol. 10, 2013.

29. K. Awan, K. N. Qureshi, and M. Mehwish, Wireless body area networks routing protocols: A review, Indonesian Journal of Electrical Engineering Computer Science, vol. 4, 2016.

30. Wang, H., Fei, B.: A modified fuzzy C-means classification method using a multiscale diffusion filtering scheme. Med. Image Anal. 13(2), 193-202 (2009)

Publisher's Note Springer Nature remains neutral with regard to jurisdictional claims in published maps and institutional affiliations. 